\title{
PSA testing for men at average risk of prostate cancer
}

\author{
Bruce K Armstrong ${ }^{a, b}$, Michael J Barry ${ }^{c, d}$, Mark Frydenberge,f, \\ Robert A Gardinerg,h,i,j,k, Ian Haines ${ }^{l, m}$ and Stacy M Cartern,o
}

a School of Population Health, University of Western Australia, Perth

b Sydney School of Public Health, University of Sydney, NSW, Australia

c General Medicine Division, Massachusetts General Hospital, Boston, US

d Department of Medicine, Harvard Medical School, Boston, MA, US

e Urological Society of Australia and New Zealand, Sydney, NSW, Australia

${ }^{\dagger}$ Department of Surgery, Faculty of Medicine, Monash University, Melbourne, Vic, Australia

g Faculty of Medicine, University of Queensland, Brisbane, Australia

${ }^{\mathrm{h}}$ Centre for Clinical Research, University of Queensland,

Brisbane, Australia

Royal Brisbane and Women's Hospital, Qld, Australia
School of Medical and Health Sciences, Edith Cowan University, Perth, WA, Australia

k Griffith University, Brisbane, Qld, Australia

I Cabrini Haematology and Oncology Centre, Cabrini Hospital, Melbourne, Vic, Australia

m AMREP Department of Medicine, Faculty of Medicine, Monash University, Melbourne, Vic, Australia

${ }^{n}$ Centre for Values, Ethics and the Law in Medicine, University of Sydney, NSW, Australia

- Corresponding author: stacy.carter@sydney.edu.au

\section{Article history}

Publication date: July 2017

Armstrong BK, Barry MJ, Frydenberg M, Gardiner RA, Haines I, Carter SM.

PSA testing for men at average risk of prostate cancer. Public Health Res Pract. 2017;27(3):e2731721. https://doi.org/10.17061/phrp2731721

\section{Key points}

- There is no formal prostate cancer screening program, but prostate-specific antigen (PSA) testing is common in Australia and some other jurisdictions and there are NHMRC approved Australian guidelines for PSA testing

- During the past 5 years, new evidence and new guidance have been produced regarding PSA testing of men at average risk of prostate cancer

- Considerable uncertainty and disagreement remain about what the evidence shows, and about whether asymptomatic men should be tested

- A commonly proposed solution is to ensure that men are well informed before they decide whether to test, but the complexity, uncertainty and very different outcomes being weighed up suggest that this would require considerable support

\begin{abstract}
Prostate-specific antigen (PSA) testing of men at normal risk of prostate cancer is one of the most contested issues in cancer screening. There is no formal screening program, but testing is common - arguably a practice that ran ahead of the evidence. Public and professional communication about PSA screening has been highly varied and potentially confusing for practitioners and patients alike.
\end{abstract}

There has been much research and policy activity relating to PSA testing in recent years. Landmark randomised controlled trials have been reported; authorities - including the 2013 Prostate Cancer World Congress, the Prostate Cancer Foundation of Australia, Cancer Council Australia, and the National Health and Medical Research Council - have made or endorsed public statements and/or issued clinical practice guidelines; and the US Preventive Services Task Force is revising its recommendations. But disagreement continues.

The contention is partly over what the new evidence means. It is also a result of different valuing and prioritisation of outcomes that are hard to compare: prostate cancer deaths prevented (a small and disputed number); prevention of metastatic disease (somewhat more common); and side-effects of treatment such as incontinence, impotence and bowel trouble (more common again). A sizeable proportion of men diagnosed through PSA testing (somewhere between 20\% and $50 \%$ ) would never have had prostate cancer symptoms sufficient to prompt investigation; many of these men are older, with competing comorbidities. It is a complex picture. 
Below are four viewpoints from expert participants in the evolving debate, commissioned for this cancer screening themed issue of Public Health Research \& Practice. We asked the authors to respond to the challenge of PSA testing of asymptomatic, normal-risk men. They raise important considerations: uncertainty, harms, the trustworthiness and interpretation of the evidence, cost (e.g. of using multiparametric magnetic resonance imaging to triage patients with elevated PSA), a likely bias towards intervention (particularly for cancer), and the potential to limit harm by treating more conservatively (although this may not occur consistently). They provide important insights, and disagree on some issues, but generally concur that men should decide for themselves whether to be tested. It seems reasonable to support men's autonomy to make their own decisions based on their own values. However, the support men might require to decide is likely to be considerable, and this needs to be taken seriously in policy making.

\section{View from a cancer epidemiologist - by Bruce K Armstrong}

That early detection of disease is good is held as true by most health professionals and most people. It is not surprising, therefore, that early detection action runs ahead of evidence of early detection benefit. This is true of all national screening programs for cancers in Australia - cervical cancer, breast cancer and colorectal cancer and it is true of prostate cancer, among others, for which there is no formal national program. The urges of clinicians to find cancer early and of people to avoid death from the 'big C' are so great that it is also not surprising that cancer screening without evidence of benefit persists, despite evidence or a strong presumption of harm. The principal harms are investigation and sometimes treatment based on a false-positive test; clinical delay and perhaps poorer outcomes after a false-negative test; and detection, diagnosis and treatment of cancers that would otherwise have never affected life or health (overdiagnosis).

How should public health policy tackle prostate-specific antigen (PSA) testing for the early detection of prostate cancer? How do we proceed when there is uncertain benefit and certain harm, when up to $21 \%$ of Australian men aged 45-74 participate in screening each year and may benefit, and 19\% aged over 74 also participate and probably won't? Ban it? Regulate it? Or do nothing? (Note: these percentages were calculated from data on claims for PSA tests [item number 66655] processed by Medicare Australia in 2015-16 [medicarestatistics.humanservices. gov.au/statistics] and Australian Bureau of Statistics tables of estimated resident population by age and sex at 30 June 2015 and 30 June 2016 [ABS report series 3101.0 Australian Demographic Statistics], with the value 7.5 added to each calculated percentage to correct for the effect of Medicare's 'episode coning' policy on recording of pathology items claimed for.)

Faced with these choices and the lack of other likely action, Cancer Council Australia (CCA) and the Prostate Cancer Foundation of Australia (PCFA) chose to 'regulate' by developing clinical practice guidelines for PSA testing and early management of test-detected prostate cancer. These nonbinding guidelines aimed "to maximise the benefits, if there are benefits, and minimise

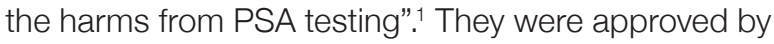
Australia's National Health and Medical Research Council on 2 November 2015. ${ }^{1}$ Early management was included because early management decisions can influence the realisation of assumed benefits and known harms of PSA testing.

The PCFA and CCA expert advisory panel made two important early decisions: that a national PSA testing program akin to the national breast cancer screening program (for example) would not be recommended, and that the reported results of the European Randomized Study of Screening for Prostate Cancer (ERSPC) would provide the evidence base for its PSA testing recommendations. The ERSPC is the largest study addressing PSA test efficacy, and one of the two largest, and the most recent, to report prostate cancer mortality reduction in PSA-tested, average-risk men. This was a necessary decision because only the ERSPC or statistical models based on its results, rightly or wrongly, can inform recommendations about the age range for, and frequency of, PSA testing, and the PSA level above which further investigation is recommended.

The guidelines make no recommendation about whether men should be routinely offered PSA testing. Their key testing recommendation is: "For men at average risk of prostate cancer who have been informed of the benefits and harms of testing and who decide to undergo regular testing for prostate cancer, offer PSA testing every 2 years from age 50 to age 69, and offer further investigation if total PSA is greater than $3.0 \mathrm{ng} / \mathrm{mL}$ ". Very importantly, they also recommend that the responsible clinical practitioner "offer evidence-based decisional support to men considering whether or not to have a PSA test, including the opportunity to discuss the benefits and harms of PSA testing before making the decision". Guidance is given as to how the PSA testing recommendation might be modified for men at higher than average risk of prostate cancer, and recommendations are made on management of men who have a PSA $>3.0 \mathrm{ng} / \mathrm{mL}$ or a biopsy diagnosis of prostate cancer, aiming at all times to obtain a balance between the benefits hoped for and the possible harms.

There is, as yet, no formal plan to evaluate implementation of the guidelines in practice or whether the assumed benefits of PSA testing are occurring, or whether the benefits are even increasing and harms decreasing. 
We might reasonably hope to see a fall in Australia's very high prostate cancer incidence rates following guideline implementation because of a fall in prostate cancer overdiagnosis due to the recommended narrower age range for, and lower frequency of, PSA testing than has been common in recent Australian practice. Because of the lack of any recommendation for or against PSA testing, and PSA's uncertain screening efficacy, any prediction of the prostate cancer mortality trend would be pure guesswork.

\section{View from the United States - by Michael J Barry}

There is a lot not to like about screening for prostate cancer with the PSA test. In the ERSPC, arguably the highest-quality trial, although not without its faults ${ }^{2}$, benefits were modest. After 13 years, 1.28 fewer prostate cancer deaths per 1000 men were observed with screening, with no decrease in overall mortality. ${ }^{3}$ Heterogeneity among ERSPC countries in screening protocols, treatments and outcomes has raised questions about the magnitude of benefit actually attributable to PSA testing. ${ }^{4}$ On the other hand, the harms of screening are relatively high, with 31 additional cases of prostate cancer needing to be detected per prostate cancer death prevented. ${ }^{3}$ Men are not used to thinking of PSA screening as 'causing' cancer, but, for practical purposes, the substantially higher incidence with screening means just that. The higher incidence reflects more prostate biopsies, with short-term complications of haematuria, haematochezia, haematospermia and infections. The main harms, however, come from the treatment of men found to have prostate cancer, including substantial risks of erectile dysfunction and incontinence. Most of these cancers are never destined to cause morbidity or mortality ${ }^{5}$, and the men who harbour them can only be harmed by early detection. Finally, at least in relation to practices in the US, PSA screening is not cost-effective. ${ }^{6}$

That all sounds like a pretty good argument for not screening, right? Perhaps. However, the absolute benefit from prostate cancer screening, if the ERSPC estimate is correct, is not dissimilar to the benefit of screening for breast cancer, and reducing overall mortality may be too high a bar for any screening test. Modelling strategies, and the reduction in metastases seen in the ERSPC, suggest that the absolute benefit may be greater over a longer time. Strategies being considered for maintaining most of the benefits while reducing the harms include testing less frequently (the screening interval in the ERSPC was 2-4 years), higher biopsy thresholds and, most importantly, active surveillance for men with low-risk cancers. These strategies to mitigate harm are not perfect and will come at the price of slightly higher risks of bad outcomes. For example, in the ProtecT study, active monitoring had the same cancer-specific mortality as surgery or radiation over 10 years (about 1\%) but a higher risk of metastases (6\% versus 3\%). ${ }^{7}$ Whether these strategies, which may make screening cost-effective ${ }^{6}$, will prove acceptable to most clinicians and patients remains to be seen. The most important reason for not rejecting PSA screening is that there is variation in how men see the trade-offs between possible benefits and harms. When men were fully informed about the trade-offs in a decision making process including a patient decision aid, and could answer key knowledge questions accurately, about a third still wanted a PSA test. ${ }^{8}$ Key points to cover in a conversation between a man and a clinician have been previously reviewed. ${ }^{5}$

Some health systems will reasonably decide not to make PSA screening generally available because their limited resources can be used more effectively in other ways. However, if PSA screening is available, letting informed men help decide whether a PSA test is right for them seems the most patient-centred way forward. After all, they are the ones who must live with the consequences of the decision.

\section{View from urologists - by Mark Frydenberg and Robert A Gardiner}

Before considering selective screening for prostate cancer, the question to be asked is whether a diagnosis will benefit the patient. The answer can only be determined by establishing whether the patient accepts the risks and potential side-effects of the diagnostic and treatment processes, appreciates the likelihood of cancer (if present) to affect his health and wellbeing, and can make a value judgement about what is really important to him. Consequently, there is no place for mass population or socalled opportunistic PSA screening.

The large majority of men investigated for prostate cancer are asymptomatic and do not have suspected malignancy based on a digital rectal examination. Because of the long natural history of most tumours, a 7-10-year life expectancy following treatment (and therefore diagnosis) is considered warranted before considering PSA testing. Pertinently, many patients have significant or latent comorbidities that will significantly affect their survival within a decade, so they will not live long enough to achieve a survival benefit. ${ }^{9-12}$

Serious health problems are not uncommon in middleaged and elderly males, along with lifestyle-related factors such as smoking and obesity, which have yet to overtly affect morbidity and mortality. A poor appreciation of individual life expectancy is not just limited to patients, as many clinicians are also overoptimistic and give patients 'the benefit of the doubt' when recommending investigations and treatments. ${ }^{12}$ To introduce some objectivity to overall patient prognosis, life expectancy tables ${ }^{13,14}$ may be helpful, although they are population based and do not take into account an individual's comorbidities or sociodemographic factors.

Selective screening involves identifying men who are at risk of developing clinically significant prostate cancer, and are likely to benefit from a prostate cancer diagnosis and 
therefore PSA testing. As we have previously reported ${ }^{15}$, a family history (particularly in first-degree relatives) is well recognised to predispose to a future diagnosis of prostate cancer, but a PSA $>1.5 \mathrm{ng} / \mathrm{mL}$ for men aged less than 50 years is regarded as even more predictive than either family history or ethnicity. ${ }^{16}$ Hereditary prostate cancers occur more commonly than any other tumour diagnosed - on average, detected 6 years earlier than for sporadic cancer. ${ }^{17}$ Patients with a family history of germ-line mutations in the family-susceptibility genes BRCA1 and BRCA2 have a significantly increased risk of developing this malignancy, tend to present at a younger age, and tend to have more aggressive disease and poorer survival outcomes. ${ }^{18-22}$

Increasingly, multiparametric magnetic resonance imaging (mpMRI) is being used to triage patients with an elevated PSA. A combination of anatomical (T2-weighted) images with at least two of the three functional MRI parameters (diffusion-weighted imaging, dynamic contrastenhanced imaging and spectroscopy) has been estimated to identify approximately $90 \%$ of moderate- to high-risk lesions, but is less reliable for detecting small $(<0.5$ cubic centimetres) and lower risk tumours. ${ }^{23-25}$ Limitations to using mpMRI are the level of accuracy of MRI interpretation and cost. However, until a cheaper and comparably accurate diagnostic test replaces PSA testing, the combination of PSA testing and mpMRI will remain the initial investigation of choice before biopsy.

It is easy to overlook or underestimate the quality-oflife impacts, which range from the anguish of possibly harbouring a malignancy to the uncommon but potentially devastating effects of infection associated with a biopsy, to the side-effects of the various treatments. Some men may rather risk a cancer spreading (especially in a cancer of lower histological grade) and remain untreated than risk losing their sexual or urinary abilities, in addition to other changes in bodily function that can affect social confidence and self-esteem. Consequently, it is imperative at the outset to evaluate and respect decisions made with respect to quality of life ${ }^{26}$ when deciding whether to test for prostate cancer.

\section{View from an oncologist - by lan Haines}

In 2012, the US Preventive Services Task Force (USPSTF) downgraded its recommendation for screening using PSA testing from $\mathrm{C}$ to $\mathrm{D} .{ }^{27}$ Since that advice, the muchanticipated ProtecT study ${ }^{28}$ has been published. It randomised 1643 men equally between radical surgery, radical radiation and active monitoring, and reported results at 10 years. It revealed that only $1 \%$ of men with early-stage prostate cancer died of their disease in the first 10 years after diagnosis, irrespective of treatment and the usual prognostic factors.

Although treatment achieved a significant reduction in local disease progression and metastases, the lack of a significant survival benefit for radical surgery over active monitoring confirms the results of the Prostate Cancer Intervention Versus Observation Trial (PIVOT), the only other randomised trial done in the era of PSA screening tests. ${ }^{29}$ It found no significant prostate cancer-specific mortality or overall survival benefit for radical surgery over active monitoring at 12 years, confirmed at 19.5 years follow-up. ${ }^{30}$ PIVOT showed an absolute risk reduction of 5.5\% (95\% confidence interval [Cl] -1.5, 12.4\%; $p=0.06)$ for all-cause mortality $(61.3 \%$ in patients treated with prostatectomy vs $66.8 \%$ in patients who had active monitoring) and an absolute risk reduction of $4 \%(95 \% \mathrm{Cl}-0.2 \%, 8.3 \%$; $p=0.06)$ for prostate cancer-specific mortality $(7.4 \%$ in patients treated with prostatectomy vs $11.4 \%$ in patients who had active monitoring). Although surgery reduced the need for treatment of progressive disease, there were increased treatment-related long-term complications such as urinary incontinence and erectile dysfunction. The dissemination of these results to every man over 40 will aid their informed decision making about PSA testing, and may reduce the overdiagnosis, overtreatment, and significant and immediate physical and psychological harms caused to many of them. ${ }^{31}$

One of the reasons for this widespread testing has been that one of the two large randomised studies of screening for prostate cancer with PSA, the ERSPC ${ }^{3}$, showed a small survival benefit for screening. However, it has been suggested by the chief medical and scientific officer of the American Cancer Society that this study has flaws and unintended biases, such as a large disparity between primary treatment with androgen deprivation monotherapy given to similar-risk patients in each arm. ${ }^{32}$ The call for an independent review of the mortality data was repeated in a recent review. ${ }^{33}$ Conversely, the other major randomised screening study ${ }^{34}$, which showed no benefit for screening, was recently found to have major contamination of the control arm, in which $90 \%$ of men had a PSA test during the study period. ${ }^{35}$ This makes its results less reliable, although it is important to note that there were still 22\% more cancers diagnosed in the screening arm after 2 years, with no associated survival benefit.

Despite the efforts of many urologists to reduce overdiagnosis and overtreatment, some urology groups suggest that all men over 40 should consider a screening PSA test ${ }^{36}$, even when we know that $24.4 \%$ of men with a 'normal' PSA are diagnosed with prostate cancer when they have a biopsy ${ }^{37}$ and it is a common finding in this age group at autopsy. ${ }^{38}$ Many are advised to consider radical treatment, with all the possible adverse consequences, for a disease that kills 1 in 7 (2-3\% of all men) at a median age of $82.4^{14}$ and for which there is still no proven survival benefit for radical intervention.

This is poor public health policy. The USPSTF should not reverse its previous advice. ${ }^{39}$

\section{Competing interests}

BA's then-employer, the Sax Institute, was reimbursed by the PCFA for remunerated time spent advising the systematic evidence review team for the PSA testing 
guideline and on writing parts of the guideline document. BA chairs the PCFA's Research Advisory Committee and is an ex officio member of its National Board. BA receives no remuneration for either of these memberships, but all expenses for attending meetings are paid for by the PCFA, and he attends Committee and Board dinners hosted by the PCFA. BA is an Associate Investigator of a PCFA randomised controlled trial of vitamin $D$ in the prevention of progression of prostate cancer managed by active surveillance. He was one of the initiators of the process to develop an Australian guideline for PSA testing and the early management of test-detected prostate cancer, and was a member of the expert advisory panel that oversaw development of the guideline and agreed on its final text.

$M B$ is a member of the USPSTF. His viewpoint does not necessarily represent the views and policies of the USPSTF. MB received salary support from the nonprofit organisation Healthwise as Chief Science Officer, and Healthwise provided grants to Massachusetts General Hospital for MB's prostate-related and shared decision making research.

$R G$ is a member of the Andrology Australia Advisory Board and is involved in NHMRC- and Movembersupported research into management of men with prostate cancer. RG and MF were members of the expert advisory panel for the NHMRC-approved Australian guidelines for PSA testing.

$\mathrm{IH}$ is a consultant oncologist who sees men in paid consultations who want second opinions about how to proceed with their PSA or biopsy results.

\section{Author contributions}

$\mathrm{BA}$ is the sole author of the section from a cancer epidemiologist. $\mathrm{MB}$ is the sole author of the section from the US. RG and MF worked together to produce the section from urologists. $\mathrm{IH}$ is the sole author of the section from an oncologist. SC wrote the abstract and key points, and collated and approved the final manuscript.

\section{References}

1. Prostate Cancer Foundation of Australia, Cancer Council Australia. PSA testing and early management of test-detected prostate cancer: clinical practice guidelines. Sydney: Prostate Cancer Foundation of Australia and Cancer Council Australia; 2016 [cited 2017 Jun 27]. Available from: wiki.cancer.org.au/ australiawiki/images/1/1b/PSA_Testing_and_Early_ Management_of_Test-detected_Prostate_Cancer_-Clinical_practice_guidelines_-_Nov15.pdf

2. Brawley OW. Prostate cancer screening: biases and the need for consensus. J Natl Cancer Inst. 2013;105(20):1522-4.

3. Schröder FH, Hugosson J, Roobol MJ, Tammela TL, Zappa M, Nelen V, et al. Screening and prostate cancer mortality: results of the European Randomised Study of Screening for Prostate Cancer (ERSPC) at 13 years of follow-up. Lancet. 2014;384(9959):2027-35.
4. Auvinen A, Moss SM, Tammela TL, Taari K, Roobol MJ, Schroder FH, et al. Absolute effect of prostate cancer screening: balance of benefits and harms by center within the European Randomized Study of Prostate Cancer Screening. Clin Cancer Res. 2016;22(1):243-9.

5. Hayes JH, Barry MJ. Screening for prostate cancer with the prostate-specific antigen test: a review of current evidence. JAMA. 2014; 311(11):1143-9.

6. Roth JA, Gulati R, Gore JL, Cooperberg MR, Etzioni R. Economic analysis of prostate-specific antigen screening and selective treatment strategies. JAMA Oncol. 2016;2(27):890-8.

7. Hamdy FC, Donovan JL, Lane JA, Mason M, Metcalfe C, Holding P, et al. 10-year outcomes after monitoring, surgery, or radiotherapy for localized prostate cancer. N Engl J Med. 2016;375(15):1415-24.

8. Barry MJ, Wexler RM, Brackett CD, Sepucha KR, Simmons LH, Gerstein BS, et al. Responses to a decision aid on prostate cancer screening in primary care practices. Am J Prev Med. 2015;49(4):520-5.

9. National Health and Medical Research Council. Prostatespecific antigen (PSA) testing in asymptomatic men. Canberra: NHMRC; 2014 [cited 2017 Jun 27]. Available from: www.nhmrc.gov.au/health-topics/testing-prostatecancer/prostate-specific-antigen-psa-testing-expertadvisory-group

10. Heidenreich A, Bastian PJ, Bellmunt J, Bolla M, Joniau S, van der Kwast $\mathrm{T}$, et al. EAU guidelines on prostate cancer. Part 1: screening, diagnosis, and local treatment with curative intent-update, 2013. Eur Urol. 2014;65(1):124-37.

11. Basch E, Oliver TK, Vickers A, Thompson I, Kantoff P, Pames $\mathrm{H}$, et al. Screening for prostate cancer with prostate-specific antigen testing: American Society of Clinical Oncology provisional clinical opinion. J Clin Oncol. 2012;30(24):3020-5.

12. Walz J, Gallina A, Saad F, Montorsi F, Perrotte P, Shariat SF, et al. A nomogram predicting 10-year life expectancy in candidates for radical prostatectomy or radiotherapy for prostate cancer. J Clin Oncol. 2007;25(24):3576-81.

13. Australian Bureau of Statistics. Canberra: Australian Bureau of Statistics; 2017. Life expectancy and deaths hit historic highs; 2015 Nov 12 [cited 2017 Jun 27]; [about 4 screens]. Available from: www.abs.gov.au/AUSSTATS/ abs@.nst/Previousproducts/3302.0Media\%20Release1201 4?opendocument\&tabname $=$ Summary $\&$ prodno $=3302$.0\&is sue $=2014 \&$ num $=\&$ view

14. Australian Bureau of Statistics. Canberra: Australian Bureau of Statistics; 2017. Deaths, Australia, 2014; 2015 Nov 12 [cited 2017 Jun 27]; [about 4 screens]. Available from: www.abs.gov.au/AUSSTATS/abs@.nsf/ Previousproducts/3302.0Main\%20Features32014?opendo cument\&tabname $=$ Summary $\&$ prodno $=3302$.0\&issue $=2014$ \&num $=\&$ view $=$ 
15. Gardiner RA, Chambers SK, Williams SG, Yaxley J, Samaratunga $\mathrm{H}$, Frydenberg M. Prostate cancer part one: detection. Chapter $10 \mathrm{In}$ : Endotext.com your endocrine source, 2014. In: De Groot LJ, Beck-Peccoz P, Chrousos G, Dungan K, Grossman A, Hershman JM, et al, editors. South Dartmouth (MA): MDText.com, Inc.; 20002014 [cited 2017 Jun 29]. Available from: www.endotext. org/chapter/prostate-cancer/

16. Vertosick EA, Poon BY, Vickers AJ. Relative value of race, family history and prostate specific antigen as indications for early initiation of prostate cancer screening. J Urol. 2014;192(3):724-9.

17. Bratt $\mathrm{O}$. What the urologist should know about hereditary predisposition to prostate cancer. BJU Int. 2007;99(4):7437.

18. Agalliu I, Karlins E, Kwon EM, Iwasaki LM, Diamond A, Ostrander EA, Stanford JL. Rare germline mutations in the BRCA2 gene are associated with early-onset prostate cancer. Br J Cancer. 2007;97(6):826-31.

19. Ford D, Easton DF, Bishop DT, Narod SA, Goldgar DE. Risks of cancer in BRCA1-mutation carriers. Breast Cancer Linkage Consortium. Lancet. 1994;343(8899):692-5.

20. Thompson D, Easton DF, Breast Cancer Linkage Consortium. Cancer incidence in BRCA1 mutation carriers. J Natl Cancer Inst. 2002;94(18):1358-65.

21. Dobson R. Prostate cancer patients with BRCA2 mutation face poor survival. BMJ. 2008;337:a705.

22. Tryggvadóttir L, Vidarsdóttir L, Thorgeirsson T, Jonasson JG, Olafsdottir GH, Rafnar T, et al. Prostate cancer progression and survival in BRCA2 mutation carriers. J Natl Cancer Inst. 2007;99(12):929-35.

23. Thompson J, Lawrentschuk N, Frydenberg M, Thompson L, Stricker P; USANZ. The role of magnetic resonance imaging in the diagnosis and management of prostate cancer. BJU Int. 2013;112 Suppl 2:6-20.

24. Thompson JE, Moses D, Shnier R, Brenner P, Delprado W, Ponsky $\mathrm{L}$, et al. Multiparametric magnetic resonance imaging guided diagnostic biopsy detects significant prostate cancer and could reduce unnecessary biopsies and over detection: a prospective study. J Urol. 2014;192(1):67-74.

25. Ahmed HU, Bosaily A E-S, Brown LC, Gabe R, Kaplan R, Parmar MK, et al. Diagnostic accuracy of multiparametric MRI and TRUS biopsy in prostate cancer (PROMIS): a paired validating confirmatory study. Lancet. 2017;389(10071):815-22.

26. O'Connor AM, Rostom A, Fiset V, Tetroe J, Entwhistle V, Lllewellyn-Thomas $\mathrm{H}$, et al. Decision aids for patients facing health treatment or screening decisions: systematic review. BMJ. 1999;319:731-4.
27. US Preventive Services Task Force. Rockville, MD: USPST; 2017. Prostate cancer: screening; 2012 May [cited 2017 Jun 27]; [about 2 screens]. Available from: www. uspreventiveservicestaskforce.org/Page/Document/ UpdateSummaryFinal/prostate-cancer-screening

28. Hamdy FC, Donovan JL, Lane JA, Mason M, Metcalfe C, Holding P, et al. 10-year outcomes after monitoring, surgery, or radiotherapy for localized prostate cancer. N Engl J Med. 2016;375(15):1415-24.

29. Wilt TJ, Brawer MK, Jones KM, Barry MJ, Aronson WJ, Fox S, et al. Radical prostatectomy versus observation for localized prostate cancer. N Engl J Med. 2012;367(3):203-13.

30. Wilt T, Jones K, Barry M, Andriole G, Culkin D, Wheeler T, et al. Follow-up of prostatectomy versus observation for early prostate cancer. New Eng J Med. 2017;377(2):132-42.

31. Donovan JL, Hamdy FC, Lane JA, Mason M, Metcalfe C, Walsh E, et al. Patient-reported outcomes after monitoring, surgery, or radiotherapy for prostate cancer. N Engl J Med. 2016;375(15):1425-37.

32. Brawley OW. Prostate cancer screening: biases and the need for consensus. J Natl Cancer Inst. 2013;105(20):1522-4.

33. Pinsky PF, Prorok PC, Kramer BS. Prostate cancer screening - a perspective on the current state of the evidence. N Engl J Med. 2017; 376(13):1285-9.

34. Andriole GL, Crawford ED, Grubb RL III, Buys SS, Chia D, Church TR, et al. Mortality results from a randomized prostate-cancer screening trial. N Engl J Med. 2009;360(13):1310-19.

35. Shoag JE and Hu JC. Reevaluating PSA testing rates in the PLCO trial. N Engl J Med. 2016;374(18)1795-6.

36. Murphy DG, Ahlering T, Catalona WJ, Crowe H, Crowe J, Clarke N, et al. The Melbourne consensus statement on the early detection of prostate cancer. BJU Int. 2014;113(2):186-8.

37. Thompson IM, Goodman PJ, Tangen CM, Lucia MS, Miller GJ, Ford LG, et al. The influence of finasteride on the development of prostate cancer. N Engl $\mathrm{J}$ Med. 2003;349(3):215-24.

38. Haas GP, Delongchamps N, Brawley OW, Wang CY, de la Roza G. The worldwide epidemiology of prostate cancer: perspectives from autopsy studies. Can J Urol. 2008;15(1):3866-71.

39. Bibbins-Domingo K, Grossman DC, Curry SJ. The US Preventive Services Task Force 2017 draft recommendation statement on screening for prostate cancer. An invitation to review and comment. JAMA. 2017; 317(19):1949-50.

\section{Copyright: (c) (i)(5) (2)}

(C) 2017 Armstrong et al. This article is licensed under the Creative Commons Attribution-NonCommercial-ShareAlike 4.0 International Licence, which allows others to redistribute, adapt and share this work non-commercially provided they attribute the work and any adapted version of it is distributed under the same Creative Commons licence terms. See: www.creativecommons.org/licenses/by-nc-sa/4.0/ 